\title{
UV Spectrophotometric Method for Determination of the Dissolution Profile of Rivaroxaban
}

\author{
Mustafa Çelebier ${ }^{1}$, Mustafa Sinan Kaynak², \\ Sacide Altınö ${ }^{1, *}$, and Selma Sahin ${ }^{3}$ \\ ${ }^{1}$ Hacettepe University, Faculty of Pharmacy, Department of Analytical Chemistry 06100 Ankara, Turkey \\ 2'Inonü University, Faculty of Pharmacy, Department of Pharmaceutical Technology, 44280, Malatya, Turkey \\ ${ }^{3}$ Hacettepe University, Faculty of Pharmacy, Department of Pharmaceutical Technology, 06100, Ankara, Turkey
}

\begin{abstract}
Rivaroxaban is an oral anticoagulant that is the first available orally active direct Factor Xa inhibitor. In this study, a UV spectrophotometric method was developed for the determination of rivaroxaban content in pharmaceutical formulations and the amount of rivaroxaban released in tablet dissolution studies. The dissolution profile of rivaroxaban was successfully determined with the validated method.
\end{abstract}

KEYWORDS: Rivaroxaban; UV spectroscopy; tablet dissolution, validation.

\section{INTRODUCTION}

$n$ vitro testing of drug release from pharmaceutical dosage forms is a common step of routine quality control as well as an initial phase of the product development process. In vitro dissolution testing of oral dosage forms measures the dissolution rate of drug substance going from the solid state into solution per unit time under standardized conditions (1,2). Dissolution testing has emerged as a very important tool in the generic pharmaceutical industry. It is widely used in formulation development, in monitoring the manufacturing process, and as a quality control test. It can also be used to predict the in vivo performance of certain products (3). In addition, dissolution testing provides valuable information on the physicochemical stability of the product $(4,5)$. Since, the release rate of the drug is unknown and its amount in the analyzed samples may vary in an unpredictable manner, it is important to choose a method where these components do not interfere with the analysis of the active substance. UV spectrophotometry is widely used for the quantitative analysis of the active substances in dissolution test samples (6-8). In this study, a UV spectrophotometric method was developed for the determination of the release of rivaroxaban (Figure 1), a new anticoagulant, from a commercially available tablet dosage form (Xarelto 10-mg).

According to the literature $(9,10)$, there are two different HPLC methods available for the determination of rivaroxaban in tablets. In the present study, a UV spectrophotometric method was developed to determine the rivaroxaban content in pharmaceutical formulations and the amount released from rivaroxaban tablets in dissolution studies. The suitability of the method was confirmed by a validation processes approved by $\mathrm{ICH}$ guidelines. The results show that the dissolution profile of rivaroxaban

${ }^{*}$ Corresponding author. and the content of rivaroxaban in tablet dosage forms were successfully determined using this UV spectrophotometric method. To our knowledge, this method is the only reported UV spectrophotometric method for the quantification of rivaroxaban in pharmaceutical dosage forms and the determination of the released amount in dissolution medium.

\section{EXPERIMENTAL}

\section{Instruments}

All spectrophotometric measurements were carried out using an Agilent 8453 model UV-vis spectrophotometer (190-1100 $\mathrm{nm}$ ) equipped with a diode array detector (DAD). The UV spectra of reference and sample solutions were recorded in $1-\mathrm{cm}$ quartz cells. The detector was set at $248 \mathrm{~nm}$.

\section{Chemicals}

All chemicals used for the preparation of stock solutions and dissolution media were of analytical grade. Standard rivaroxaban was supplied by Refik Saydam National Public Health Agency. Water obtained from a Milli-Q water system (Barnstead, USA) was used for the preparation of dissolution media. Commercially available rivaroxaban tablets (Xarelto 10-mg) were provided by Bayer Turk.

\section{Dissolution Studies}

Dissolution studies on the rivaroxaban (Xarelto 10-mg) tablets were conducted using USP Apparatus 2 (paddle method) with six replicates at $37 \pm 0.5^{\circ} \mathrm{C}$ (Sotax, Switzerland). A pH 4.5 acetate buffer containing $0.4 \%$ of sodium dodecyl sulfate (SDS) was the dissolution medium (900 $\mathrm{mL}$ ), and the the paddle rotation speed was $50 \mathrm{rpm}$. In all experiments, $2-\mathrm{mL}$ sample aliquots were withdrawn at predetermined time intervals $(0,5,10,15,20,30,40$, 50 , and $60 \mathrm{~min}$ ) and replaced with an equal volume of 


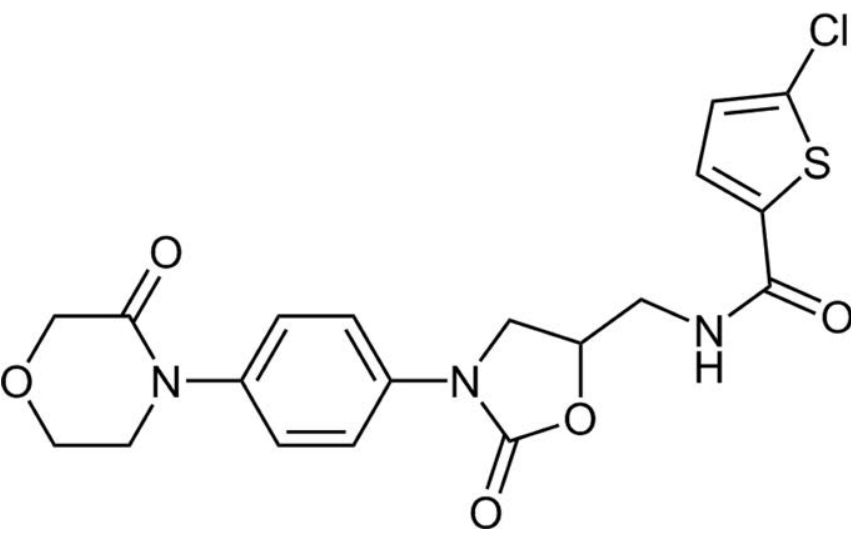

Figure 1. Chemical structure of rivaroxaban.

fresh medium to maintain a constant total volume. After the filtration of the dissolution samples using $0.44-\mu \mathrm{m}$ membrane filters, the concentrations of rivaroxaban were determined simultaneously by the proposed spectrophotometric method.

\section{Calibration Standards}

A stock standard solution of rivaroxaban $(1000 \mu \mathrm{g} / \mathrm{mL})$ was prepared by dissolving $25 \mathrm{mg}$ of compound in $25 \mathrm{~mL}$ of an acetonitrile (ACN)/water (50:50 v/v) mixture. Calibration standards $(1.0,2.0,5.0,10.0,20.0,30.0$, and $50.0 \mu \mathrm{g} /$ $\mathrm{mL}$ ) were prepared daily from the stock standard solution by appropriate dilution using dissolution buffer.

\section{Tablet Solutions}

Ten tablets were weighed to determine the mean weight and finely powdered in a mortar. An amount of powdered mass equivalent to one tablet weight was accurately weighed and transferred to a $100-\mathrm{mL}$ volumetric flask. Fifty milliliters of ACN/water $(50: 50 \mathrm{v} / \mathrm{v})$ was added, and the mixture was sonicated for $15 \mathrm{~min}$ to ensure complete extraction of drug. The flask was then brought to volume. An aliquot was filtered through a $0.45-\mu \mathrm{m}$ membrane filter. The final tablet solutions were further diluted with ACN/water (50:50 v/v) to a concentration of $10 \mu \mathrm{g} / \mathrm{mL}$ of rivaroxaban.

\section{RESULTS AND DISCUSSION}

UV spectrophotometry has widespread usage in drug analysis because of its capability for rapid analysis with good repeatability. The key point in applying UV spectrophotometry to drug analysis is ensuring that matrix components do not interfere with the active ingredient signal. The dissolution studies of rivaroxaban tablets were performed using $\mathrm{pH} 4.5$ acetate buffer containing $0.4 \%$ of sodium dodecyl sulfate (SDS) as the dissolution medium. Therefore, all calibration standards (Figure 2) and tablet samples were prepared using the dissolution medium.

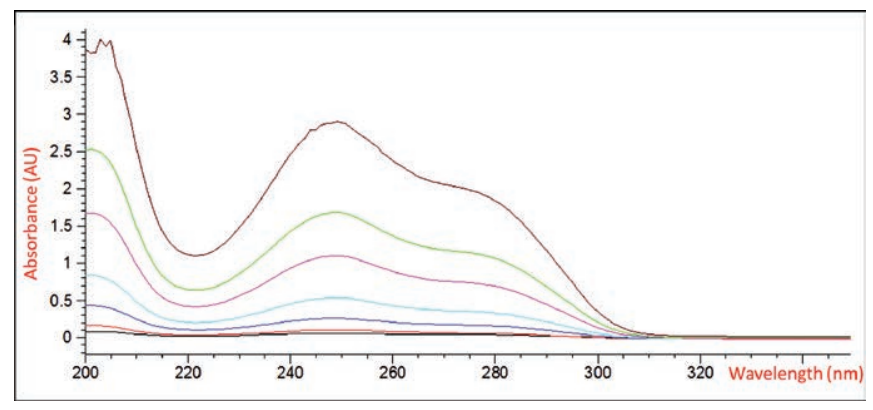

Figure 2. Spectra of standard rivaroxaban solutions under experimental conditions $(1.0-50.0 \mu \mathrm{g} / \mathrm{mL}$ ).

Table 1. Method Linearity and Sensitivity $(n=6)$

\begin{tabular}{ll}
\hline Regression equation ${ }^{a}$ & $y=(0.0584 \pm 0.0002) x-(0.0242 \pm 0.0019)$ \\
\hline Correlation coefficient $(r)$ & 0.9997 \\
\hline Linearity range $(\mu \mathrm{g} / \mathrm{mL})$ & $0.5-50$ \\
\hline $\mathrm{LOQ}(\mu \mathrm{g} / \mathrm{mL})$ & 0.5 \\
\hline $\mathrm{LOD}(\mu \mathrm{g} / \mathrm{mL})$ & 0.3 \\
\hline $\begin{array}{l}{ }^{a} y=(a \pm S E) x+(b \pm S E) \\
\text { and } S E \text { is standard error. }\end{array}$ \\
\hline
\end{tabular}

The samples obtained from content uniformity and dissolution studies were analyzed at $248 \mathrm{~nm}$; there was no interference from matrix components. The method was validated (11-13) to demonstrate precision and accuracy. The absorbance values of calibration standards at $248 \mathrm{~nm}$ were plotted against the corresponding concentrations to obtain the calibration curve. Linearity and sensitivity of the method are shown in Table 1. To demonstrate the precision and accuracy of the method, intra- and interday studies were performed, and the results were evaluated statistically (Table 2). The results show that the precision and accuracy of the method meet the acceptance criteria.

The accuracy of the method was also investigated through recovery studies. The standard addition technique was applied; a known amount of standard rivaroxaban solution was added to tablet solutions at 5.0, 10.0, and $20.0 \mu \mathrm{g} / \mathrm{mL}$, and these solutions were then analyzed. The slope of the standard addition curve $(0.0568 \pm 0.0012$, $n=6$ ) was identical to the curve of calibration standards at the selected wavelength. Figure 3 presents the spectra of standard, tablet, and blank (dissolution medium) solutions. The spectra show that the absorbance of tablet and standard solutions having the same concentration of rivaroxaban is identical at $248 \mathrm{~nm}$; there is no absorbance by the dissolution medium at this wavelength. This observation confirms that there is no interference from matrix components at $248 \mathrm{~nm}$. 
Table 2. Method Precision and Accuracy $(n=3)$

\begin{tabular}{|c|c|c|c|c|c|c|}
\hline \multirow[t]{2}{*}{ Added $(\mu \mathrm{g} / \mathrm{mL})$} & \multicolumn{3}{|c|}{ Intraday } & \multicolumn{3}{|c|}{ Interday } \\
\hline & Found ( $\mu \mathrm{g} / \mathrm{mL})$ & Precision RSD \% & Accuracy \% Bias & Found $(\mu g / m L)$ & Precision \% RSD & Accuracy \% Bias \\
\hline 2 & $2.08 \pm 0.03$ & 2.65 & 3.83 & $2.07 \pm 0.02$ & 1.96 & 3.33 \\
\hline 10 & $9.72 \pm 0.04$ & 0.78 & 2.88 & $9.71 \pm 0.08$ & 1.48 & -2.86 \\
\hline 30 & $29.83 \pm 0.07$ & 0.41 & 0.56 & $30.40 \pm 0.50$ & 2.85 & 1.33 \\
\hline $\begin{array}{ll}\text { Found: } & \text { mean } \pm \text { star } \\
\text { RSD: } & \text { Relative sta } \\
\text { Bias: } & {[(\text { Found }-A c}\end{array}$ & $\begin{array}{l}\text { rd error }(n=6) \\
\text { rd deviation } \\
\text { d) } / \text { Added }] \times 100\end{array}$ & & & & & \\
\hline
\end{tabular}

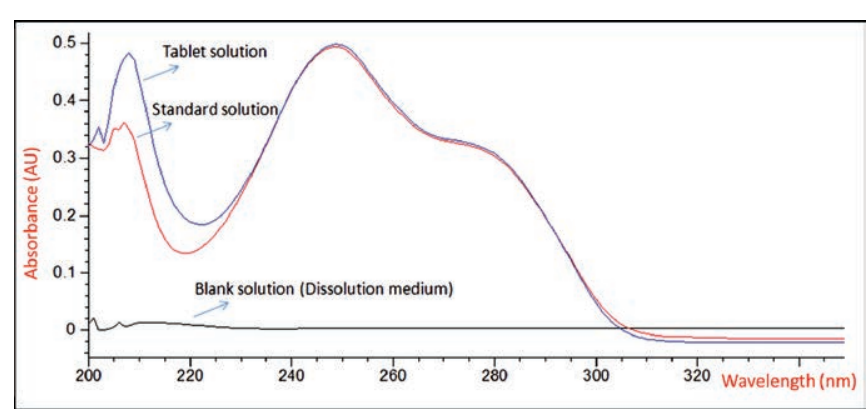

Figure 3. Spectra of standard, tablet, and blank (dissolution medium) solutions.

Determination of the rivaroxaban content in film-coated tablets (Xarelto 10-mg) was achieved by analyzing six tablet solutions prepared as described in the experimental section. The results of the assay $(n=6)$ yielded $10.24 \pm 0.14 \mathrm{mg}$ (mean $\pm \mathrm{SE}$ ) rivaroxaban corresponding to $102.38 \%$ (RSD $=3.35$ ) of the labeled claim. The sample recoveries in the formulation were in good agreement with the respective label claim, which suggests no interference associated with the formulation excipients in the analysis. In addition, the RSD values in the tablet analysis were less than $1 \%$ indicating a high degree of precision of the proposed method.

\section{In Vitro Dissolution Studies}

The average percentage of drug released within 60 min as determined by the proposed spectrophotometric method after in vitro dissolution of tablets is depicted in Figure 4 . Within $15 \mathrm{~min}, 78 \%$ of rivaroxaban was released. The dissolution profile complies with the FDA guidance (14), indicating suitability of the proposed method for the dissolution testing of rivaroxaban (15).

\section{CONCLUSIONS}

The proposed method is rapid, accurate, precise, and sensitive for the quantification of rivaroxaban from tablet dosage forms. The method relies on the use of a simple procedure with results comparable to those achieved by

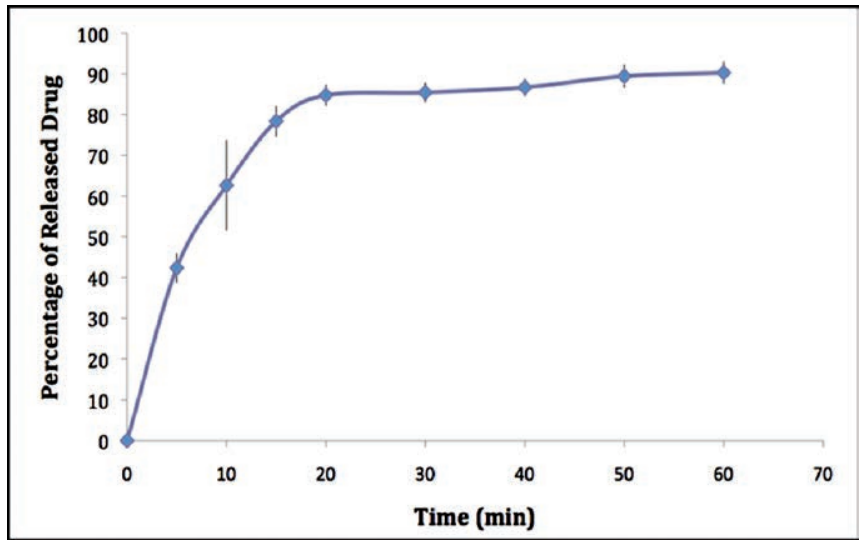

Figure 4. Percentage of rivaroxaban released from tablets (vertical lines indicate standard error).

sophisticated and expensive techniques such as HPLC. Hence, this method can be routinely employed in the quality control analysis and dissolution studies of rivaroxaban in tablets.

\section{REFERENCES}

1. Drug Release Testing. Particle Sciences Drug Development Services. http://www.particlesciences. com/services/drug-release-testing/ (accessed Oct 1, 2014).

2. Dunne, A.; Devane, J.; O'Hara, T. The Relationship Between In Vitro Drug Dissolution and In Vivo Absorption. J. R. Stat. Soc. D-Sta. 1999, 48, 125-133. DOI: 10.1111/1467-9884.00176.

3. Anand, O.; Yu, L. X.; Conner, D. P.; Davit, B. M. Dissolution Testing for Generic Drugs: An FDA Perspective. AAPS J. 2011, 13 (3), 328-35. DOI: 10.1208/ s12248-011-9272-y.

4. Mudie, D. M.; Amidon, G. L.; Amidon, G. E. Physiological Parameters for Oral Delivery and in Vitro Testing. Mol. Pharmaceutics 2010, 7 (5), 1388-1405. DOI: 10.1021/ mp100149j.

5. Abrahamsson, B. In vitro/in vivo correlations for drug dissolution. Eur. J. Pharm. Sci. 2003, 19, S16-S17.

6. Busaranon, K.; Suntornsuk, W.; Suntornsuk, L. Comparison of UV spectrophotometric method and 
high performance liquid chromatography for the analysis of flunarizine and its application for the dissolution test. J. Pharm. Biomed. Anal. 2006, 41 (1), 158-164. DOI: 10.1016/j.jpba.2005.11.008.

7. Pourmand, M. R.; Azar, M. S.; Aghavalijamaat, M. Development of validated UV spectrophotometric method for in vitro analysis of sumatriptan in pharmaceutical preparations in comparison with HPLC. Pharm. Chem. J. 2011, 44 (10), 585-589. DOI: 10.1007/s11094011-0522-1.

8. Varma, M. V.; Kaushal, A. M.; Garg, S. Rapid and selective UV spectrophotometric and RP-HPLC methods for dissolution studies of oxybutynin immediaterelease and controlled-release formulations. J. Pharm. Biomed. Anal. 2004, 36 (3), 669-674. DOI: 10.1016/j. jpba.2004.07.044.

9. Sekhar, K. C.; Vani, P. S.; Lakshmi, A. D.; Devi, Ch. L.L.; Barik, A.; Devanaboyina, N. A New Method Development and Validation for Analysis of Rivaroxaban in Formulation by RP HPLC. Res. Desk 2012, 1 (3), 24-33.

10. Satyanarayana, P. V. V.; Madhavi, A. S. RP-HPLC method development and validation for the analysis of rivaroxaban in pharmaceutical dosage forms. Int. J. Sci. Innovations Discoveries 2012, 2 (1), 226-231.

11. International Conference on Harmonisation of Technical Requirements for Registration of Pharmaceuticals for Human Use. Validation of Analytical Procedures: Text and Methodology, Q2(R1);
ICH Harmonised Tripartite Guideline: Geneva, Switzerland, 2005.

12. Taverniers, I.; De Loose, M.; Van Bockstaele, E. Trends in quality in the analytical laboratory. I. Traceability and measurement uncertainty of analytical results. TrAC Trends Anal. Chem. 2004, 23 (7), 480-490. DOI: 10.1016/ S0165-9936(04)00733-2.

13. Taverniers, I.; De Loose, M.; Van Bockstaele, E. Trends in quality in the analytical laboratory. II. Analytical method validation and quality assurance. TrAC Trends Anal. Chem. 2004, 23 (8), 535-552. DOI: 10.1016/j. trac.2004.04.001.

14. Dissolution Testing of Immediate Release Solid Oral Dosage Forms; Guidance for Industry; U.S. Department of Health and Human Services, Food and Drug Administration, Center for Drug Evaluation and Research (CDER), U.S. Government Printing Office: Washington, DC, 1997. http://www.fda.gov/downloads/Drugs/ GuidanceComplianceRegulatoryInformation/ Guidances/ucm070237.pdf (accessed Oct 1, 2014).

15. Rivaroxaban Clinical Pharmacology Review Addemdum, Clinical Pharmacology and biopharmaceutics Review; U.S. Department of Health and Human Services, Food and Drug Administration, Center for Drug Evaluation and Research (CDER), U.S. Government Printing Office: Washington, DC, 2011. http://www.accessdata.fda.gov/ drugsatfda_docs/nda/2011/2024390rig1s000ClinPhar mR.pdf (accessed Oct 1, 2014). 